\title{
Morphological and Biochemical Characterization of Diverse Strawberry Tree (Arbutus unedo L.) Genotypes from Northern Turkey
}

\author{
Halil Ibrahim Sagbas ${ }^{1}{ }^{10}$, Gulce Ilhan ${ }^{1}$, Hafida Zitouni ${ }^{2}$, Muhammad Akbar Anjum ${ }^{3}$, \\ Hafida Hanine ${ }^{2}$, Tomas Necas ${ }^{4}, *$, Ivo Ondrasek ${ }^{4}$ and Sezai Ercisli ${ }^{2}$ (D) \\ 1 Department of Horticulture, Agricultural Faculty, Ataturk University, 25240 Erzurum, Turkey; \\ hibrahimsagbas@gmail.com (H.I.S.); gulceilhan07@gmail.com (G.I.) \\ 2 Laboratory of Bioprocess and Bio-Interfaces, Faculty of Science and Technics, University Sultan Moulay \\ Slimane, BO 523, Beni-Mellal 23000, Morocco; hafida2012ziitn@gmail.com (H.Z.); \\ hafidahanina0@gmail.com (H.H.); sercisli@gmail.com (S.E.) \\ 3 Department of Horticulture, Bahauddin Zakariya University, Multan 60800, Pakistan; anjumbzu@yahoo.com \\ 4 Department of Fruit Science, Faculty of Horticulture in Lednice, Mendel University in Brno, 61300 Brno, \\ Czech Republic; ivo.ondrasek@mendelu.cz \\ * Correspondence: tomas.necas@mendelu.cz; Tel.: +420-519367244
}

Received: 15 September 2020; Accepted: 14 October 2020; Published: 16 October 2020

\begin{abstract}
Wild edible fruits have been used centuries by local people mainly for nutrition purposes and they have attracted the attention of researchers in the last decade throughout the world. Among wild edible fruits, the strawberry tree (Arbutus unedo) is widely distributed mainly in Mediterranean countries. However, the lack of information about strawberry tree genetic resources in Mediterranean countries is a major cause of its low exploitation by breeders. For a practical use and exploitation of the strawberry tree native germplasm, phenological, morphological, and biochemical characterization of the plant is essential. In this study, some important phenological, morphological, and biochemical characteristics of sixteen local strawberry tree genotypes were determined. Significant variations $(p<0.05)$ were observed in most of the investigated phenological, morphological, and biochemical traits of the genotypes. The genotypes were harvested between 2-21 November. The fruit weight and the number of fruits per cluster of the genotypes varied from 3.89 to $6.86 \mathrm{~g}$ and 3.61 to 7.94 , respectively. Vitamin $\mathrm{C}$ and total dietary fiber contents of genotypes were found quite variable, ranging from 58 to $93 \mathrm{mg} / 100 \mathrm{~g}$ and 7.04 to $12.36 \mathrm{~g} / 100 \mathrm{~g}$, respectively. The fruits of the strawberry tree include mainly fructose and glucose rather than sucrose. Local strawberry tree genotypes are found to be promising for further valorization through horticultural characteristics for breeding. Some genotypes had significantly higher fruit weight, vitamin C concentrations, total dietary fiber, and total phenolic concentration and may be good selections for producing health products.
\end{abstract}

Keywords: strawberry tree; diversity; morphology; composition

\section{Introduction}

Turkey is the world's largest fruit producer after China (24 MT), India (99 MT), Brazil (40 MT), and USA (26 MT) with 24 MT of fruit production per year [1]. Such a high fruit production is one of the main income sources in agriculture of the country. Besides cultivated fruits, the country also has very rich wild edible fruits $[2,3]$.

Compared to cultivated ones, wild edible fruit species acquire wild plasticity through the combination of both the long-term accumulation of genetic changes and the conservation of favorable survival strategies through time and they could be important for use in the future as a safeguard for 
climate change. Phenotypic plasticity, especially within wild edible fruit populations, is a mechanism that enhances plant survival $[4,5]$.

The Black Sea region of Turkey, geographically known as North Anatolia, is one of the plant biodiversity areas in Turkey. The region is divided into the western (from Edirne to Sinop), central (from Sinop to Unye), and eastern (from Unye to Georgia border) parts and phytogeographically by the region called the Euro-Siberian region dominated by Euro-Siberian elements and some Mediterranean enclaves [6,7]. The region has great potential not only for cultivated fruits such as hazelnut, kiwifruit, persimmon, blueberry, but also for wild edible fruits viz. Vaccinium, Cerasus, Fragaria, Eriobotrya, Mespilus, Morus, Cornus, Castanea, Diospyros, Arbutus, Rubus etc. that remained underexploited due to the lack of awareness of their potential, market demand, and value addition [8-10]. For centuries, these underutilized fruits are propagated by seeds and traditionally used only by local inhabitants and traded locally in the Black Sea region and the fruits are sold on local markets and roadsides as fresh, dried, or processed [11,12]. The local community commonly consumes local fruits as a functional food and nutraceutical supplement [9].

Most of the wild edible fruits in the region are generally found in forests as wild or semi-wild in homestead gardens as shrubs or tree with considerable morphological, agronomical, and biochemical variability. They are rich sources for bioactive substances as well as carbohydrates, fats, proteins, energy, vitamins-A, $B_{1}, B_{2}, B_{3}, B_{6}, B_{9}, B_{12}, C$, folic acid, and minerals- $\mathrm{Ca}, \mathrm{P}, \mathrm{Fe}$, and dietary fiber $[2,3,9,13]$. These crops have many advantages, like easier to grow and hardy in nature, producing a crop even under adverse soil and climatic conditions. So, exploitation of underutilized wild edible fruits and conservation of them can become a solution to the social problem of health and nutrition insecurity, poverty, and unemployment $[4,5]$.

Much of the underutilized fruits' genetic variability in the world has been maintained and conserved ex situ $[9,14]$. Breeding programs need a wide germplasm, with different plant and fruit characteristics that are easily determined by morphological, agronomic, biochemical, and molecular methods. Thus, to obtain information and maintain diversity on wild edible fruit species is vital for future practical use $[3,14-17]$. Among the characterization methods, morphological and biochemical characterization is preferred because it is cheaper and easier [3,18-21]. In addition, morphological and biochemical characterization provides the information's underlying conclusions on the genetic variability of the genotypes and supports the regeneration and maintenance of the genetic integrity of genotypes [22].

Strawberry trees (A. unedo and $A$. andrachne) are evergreen shrubs or small trees and in late autumn and early winter, bear red or orange colored fruits, naturally grown as a population or solitary tree in the coastal areas including Mediterranean, Aegean, Marmara, and Black Sea region in Turkey [2,6]. The A. unedo fruits are more attractive and more promising than A. andrachne. Most of the Mediterranean countries such as Algeria, Morocco, Tunisia, Turkey, Syria, Greece, Croatia, France, Portugal, and Spain have native A. unedo populations. Rural people are utilizing its fruits and leaves as a functional food and for medicinal applications to treat ailments, respectively [23]. In coastal regions in Turkey and the other Mediterranean countries, strawberry trees are the predominant, yet largely uncharacterized regarding their morphological and pomological properties. Therefore, the evaluation of local germplasm and indigenous genotypes stands as an emerging need.

There is rising interest in strawberry tree fruits in Turkey. Recently, strawberry tree fruits have stepped out from small local production to a niche product that receives appreciably high prices. Particularly, it is a unique harvested fruit in early winter. This is mainly attributed to the lack of other fleshy fruits in the market during its harvesting period at the end of autumn and beginning of winter. Overall, the strawberry tree can potentially be identified as a promising fruit for market $[6,8]$.

Wild edible strawberry trees show great morphological and biochemical diversity in growing locations in the Black Sea region in Turkey due to seed propagation for centuries. However, there is still a lack of data related to the morphological and biochemical characteristics of these fruits. Thus, the main objective of this study was to provide a comprehensive characterization of wild grown local strawberry 
tree genotypes and then to explore their potential for future cultivation conditions in orchards and as well, obtain information for their use as an innovative food ingredient.

\section{Materials and Methods}

\subsection{Plant Material}

The Black Sea region of Turkey has a large number of seed propagated strawberry tree (Arbutus unedo L.) genotypes that show great variability for most of the tree and fruit characteristics. In fact, all available strawberry tree genotypes in fields in the region are found as solitary tree genotypes (farmer selections). Traditionally, farmers left seed propagated strawberry tree genotypes in the field if they had good fruit and yield, whilst they removed genotypes with low yields and poor fruit quality. In this experiment, a total of sixteen seed propagated (un-grafted) strawberry tree genotypes naturally grown in Akcaabat district (latitude and longitude as $41.02^{\circ} \mathrm{N}$ and $39.57^{\circ} \mathrm{E}$ ) of the Trabzon province were used. The Trabzon lies on $38 \mathrm{~m}$ above sea level and the climate is warm with an average annual temperature and precipitation of $14.4{ }^{\circ} \mathrm{C}$ and $891 \mathrm{~mm}$. The strawberry tree fruits were harvested during 2017 and 2018 from the 16 seed propagated plants. In field, only one seed propagated genotype was available, thus we used only one tree per genotype. The fruit samples were collected from all parts to fully represent 16 strawberry tree genotypes and were placed into sample containers and transported to the laboratory and stored at $-80^{\circ} \mathrm{C}$ until the biochemical analysis was done. Fruit weight, peel roughness, and fruit color were determined unfrozen fruit samples immediately. The plant ages of genotypes were between 23-25 years old. For biochemical analysis, flesh (mesocarp) tissue of fruits was used. Morphological and biochemical analysis was conducted on 40 fruits (10 fruits per replicate).

\subsection{Harvest Date and Morphological Characteristics}

The fruits were harvested in the field when fully ripe, i.e., when they reached their characteristic mature color (in general, red in the strawberry tree fruits) and enough sweetness and softness to make them more palatable. A total of 40 fruits ( 10 fruits per replicate) were sampled from each genotype to determine traits such as fruit weight (g), fruit pedicel length, fruit color (flesh and peel), and fruit skin roughness. Fruit dimensions (width and length) were determined to calculate fruit shape values. Leaf dimensions, such as length and width were determined. For this, 40 leaves (10 leaves per replicate) were used. Fruit weights (g) were determined with a digital balance $( \pm 0.01 \mathrm{~g})$ (Scaltec SPB31, Scaltec Instruments $\mathrm{GmbH}$, Goettingen, Germany). Fruit and leaf dimensional characteristics were measured with a digital caliper $( \pm 0.01 \mathrm{~mm})$ (Mitutoyo 500-196-20, Mitutoya Co., Kawasaki, Japan). The fruit color was measured using a portable Minolta Chroma Meter (Minolta, Model CR-400, Tokyo, Japan), which provided CIE $\mathrm{L}^{*}, \mathrm{a}^{*}$ and $\mathrm{b}^{*}$ values [24]. A trained panel of five experts evaluated the roughness and visible peel and flesh color for each genotype. The 1 to 5 bipolar hedonic scale was used to describe peel roughness, which was rated as low, medium, and high [25].

\subsection{Extraction and Determination of Organic Acids}

For the extraction of organic acids, the procedure described by Bevilacqua and Califano [26] was followed with modifications. First, $200 \mathrm{~g}$ samples were shred and $10 \mathrm{~g}$ from each sample was transferred to centrifuge tubes. The samples were mixed with $10 \mathrm{~mL}$ of $0.009 \mathrm{~N} \mathrm{H}_{2} \mathrm{SO}_{4}$ and then homogenized with a Heidolph Silent Crusher M, Schwabach, Germany. Thereafter, the samples were mixed by gentle shaking for an hour (Heidolph Unimax 1010, Schwabach, Germany) and centrifuged at 14,000 rpm for $15 \mathrm{~min}$. The supernatant was filtered through a coarse filter paper, then twice through a $0.45 \mu \mathrm{m}$ membrane filter (Millipore Millex-HV Hydrophilic PVDF, Millipore, Burlington, MA, USA), and finally in a SEP-PAK C18 cartridge. The concentrations of organic acids were determined by HPLC using an Aminex column (HPX-87H, $300 \mathrm{~mm} \times 7.8 \mathrm{~mm}$, Bio-Rad, Hercules, CA, USA) fitted on an Agilent 1100 series HPLC G 1322 A, Pliening, Germany [26]. Organic acids were determined at 210 and $250 \mathrm{~nm}$ wavelengths. Then, $0.009 \mathrm{~N} \mathrm{H}_{2} \mathrm{SO}_{4}$ was used as the mobile phase. 


\subsection{Extraction and Determination of Sugars}

The samples were prepared following the procedure described by Melgarejo et al. [27] with small modifications. Briefly, a $10 \mathrm{~g}$ fruit sample was centrifuged at $12,000 \mathrm{rpm}$ for $2 \mathrm{~min}$. at $4{ }^{\circ} \mathrm{C}$. Then, the supernatant was filtered with a SEP-PAK C18 cartridge and transferred into a vial for analysis. The concentrations of sugars were determined by HPLC (isocratic program), with a $\mu$ Bondapak- $\mathrm{NH}_{2}$ column and a refractive index (RI) detector, and with $85 \%$ acetonitrile as the mobile phase. The quantification of the concentrations was based on standards.

\subsection{Determination of Trolox Equivalent Antioxidant Capacity (TEAC)}

For total antioxidant determination, Trolox equivalent antioxidant capacity (TEAC) assay was used [28].

\subsection{Vitamin C Determination}

A reflectometer (Reflectometer RQflex ${ }^{\circledR}$, Merck KGaA, Darmstadt, Germany) was used for fruit juice vitamin $\mathrm{C}$ measurement and the result expressed as $\mathrm{mg}$ vitamin $\mathrm{C}$ (ascorbic acid) per $100 \mathrm{~g}$ fresh fruit base [19].

\subsection{Total Phenol Determination}

The total phenolic concentration of strawberry tree fruits was determined spectrophotometrically (Mettler Toledo UV Vis, Columbus, OH, USA) described by Singleton et al. [29] and the results are expressed as $\mathrm{mg}$ gallic acid equivalents per $100 \mathrm{~g}$ of fruit.

\subsection{Total Dietary Fiber Determination}

Dietary fiber was determined and described by De Padua et al. [30].

\subsection{Crude Lipid Determination}

The AOAC [31] method was used to determine crude lipid concentration of strawberry tree fruits by using the Soxhlet extractor for $8-10 \mathrm{~h}$ to extract crude lipid. The amount of crude lipid was calculated and expressed as the percentage of crude lipid concentration.

\subsection{Extraction and Determination of Phenolic Compounds}

The phenolic compounds in strawberry tree fruits were determined following the procedure described by Rodriguez-Delgado et al. [32]. The separation of chromatographic data in an Agilent 1100 series HPLC took place in a DAD detector (Agilent, Santa Clara, CA, USA) with a $250 \mathrm{~mm} \times 4.6 \mathrm{~mm}$, $4^{\prime} \mu \mathrm{m}$ ODS column (HiChrom, Reading, UK).

\subsection{Statistical Analysis}

SPSS software was used for statistical analysis and results were evaluated with the least significant difference (LSD) method at $p<0.05$. In Tables, an average of 2 years data were given because there were no statistical differences between years. PCA was used in a two-dimensional array of eigenvectors using the DCENTER and EIGEN modules of NTSYS-pc. The relationship among traits was revealed by conducting Pearson's correlation analysis.

\section{Results}

In the present investigation, we assessed variations in morphological and biochemical characteristics among 16 strawberry tree genotypes and all phenological and morphological traits exhibited significant differences $(p<0.05)$ (Tables 1 and 2). 
Table 1. Some phenological and morphological characteristics of fruits and leaves belong to 16 strawberry tree genotypes (average of 2017-2018 years).

\begin{tabular}{|c|c|c|c|c|c|c|c|}
\hline Genotypes & Fruit Weight (g) & $\begin{array}{l}\text { The Number of } \\
\text { Fruit per Cluster }\end{array}$ & Harvest Dates & Shape Index & $\begin{array}{l}\text { Fruit Pedicel } \\
\text { Length }(\mathrm{mm})\end{array}$ & $\begin{array}{l}\text { Leaf Length } \\
(\mathrm{mm})\end{array}$ & $\begin{array}{l}\text { Leaf Width } \\
\text { (mm) }\end{array}$ \\
\hline AU-1 & $5.31^{\mathrm{c}}$ & $4.45^{\text {ef }}$ & 9 Nov. & $1.09^{\mathrm{a}}$ & $3.08^{\mathrm{cd}}$ & $46.15^{\mathrm{ab}}$ & $26.15^{\mathrm{ab}}$ \\
\hline AU-2 & $6.12^{\mathrm{ab}}$ & $3.93 \mathrm{fg}$ & 7 Nov. & $0.92^{c}$ & $3.27^{\mathrm{cd}}$ & $37.41^{b c}$ & $20.12^{b c}$ \\
\hline AU-4 & $3.89^{\mathrm{e}}$ & $6.74^{\mathrm{a}}$ & 4 Nov. & $0.96^{b c}$ & $2.93^{\mathrm{d}}$ & $33.04^{b c}$ & $14.90^{\mathrm{d}}$ \\
\hline AU-5 & $6.44^{\mathrm{ab}}$ & $3.88^{\mathrm{fg}}$ & $6 \mathrm{Nov}$ & 0.80 de & $3.56^{\mathrm{cd}}$ & $38.76^{b c}$ & $20.35^{b c}$ \\
\hline AU-6 & $4.74^{\mathrm{cd}}$ & $5.55^{\mathrm{cd}}$ & 21 Nov. & $0.78^{e}$ & $4.41^{b c}$ & $51.22^{\mathrm{a}}$ & $27.30^{\mathrm{a}}$ \\
\hline AU-9 & $4.43^{\mathrm{d}}$ & $5.40^{\mathrm{cd}}$ & 11 Nov. & $0.95^{b c}$ & $5.81^{\mathrm{a}}$ & $35.02 \mathrm{bc}$ & $18.13^{b c}$ \\
\hline AU-10 & $4.98^{\mathrm{cd}}$ & $5.20^{\mathrm{d}}$ & 18 Nov. & 0.80 de & $4.56^{\mathrm{bc}}$ & $41.89^{a b}$ & $24.56^{\mathrm{ab}}$ \\
\hline AU-11 & $6.86^{a}$ & $3.61^{g}$ & 9 Nov. & $0.96^{b c}$ & $5.07^{b}$ & $33.40^{b c}$ & $16.33^{\mathrm{cd}}$ \\
\hline AU-12 & 3.97 de & $6.33^{b}$ & 12 Nov. & $1.01^{\mathrm{ab}}$ & $4.30^{b c}$ & $41.30^{\mathrm{b}}$ & $22.44^{b}$ \\
\hline AU-13 & $6.21^{\mathrm{ab}}$ & $4.12^{\mathrm{f}}$ & 2 Nov. & $0.93^{c}$ & $5.40^{\mathrm{ab}}$ & $32.15^{c}$ & $14.76^{\mathrm{d}}$ \\
\hline AU-14 & $6.02^{b}$ & $4.03 \mathrm{fg}$ & 8 Nov. & $1.06^{\mathrm{ab}}$ & $5.15^{\mathrm{ab}}$ & $35.15^{b c}$ & $18.56^{b c}$ \\
\hline
\end{tabular}

Different letters in the same column indicate significant $(p<0.05)$ differences among genotypes.

Table 2. Some color and roughness characteristics of fruits belonging to the strawberry tree (average of 2017-2018 years).

\begin{tabular}{ccccccc}
\hline Genotypes & \multicolumn{1}{c}{$\boldsymbol{L}$} & $\boldsymbol{a}$ & Peel Color & Flesh Color & Peel Roughness \\
\hline AU-1 & $41.25^{\mathrm{b}}$ & $40.65^{\mathrm{b}}$ & $23.98^{\mathrm{b}}$ & Red & Orange & Low \\
AU-2 & $38.11^{\mathrm{bc}}$ & $38.56^{\mathrm{bc}}$ & $27.36^{\mathrm{ab}}$ & Red & Light orange & Medium \\
AU-3 & $38.86^{\mathrm{bc}}$ & $42.12^{\mathrm{ab}}$ & $27.02^{\mathrm{ab}}$ & Dark red & Dark orange & Low \\
AU-4 & $42.20^{\mathrm{ab}}$ & $37.13^{\mathrm{bc}}$ & $22.99^{\mathrm{bc}}$ & Light red & Orange & Medium \\
AU-5 & $37.56^{\mathrm{c}}$ & $43.12^{\mathrm{ab}}$ & $25.45^{\mathrm{ab}}$ & Dark red & Light orange & Low \\
AU-6 & $40.45^{\mathrm{bc}}$ & $32.23^{\mathrm{d}}$ & $20.27^{\mathrm{c}}$ & Light red & Orange & Medium \\
AU-7 & $37.77^{\mathrm{bc}}$ & $37.04^{\mathrm{bc}}$ & $21.29^{\mathrm{bc}}$ & Light red & Light orange & Low \\
AU-8 & $39.17^{\mathrm{bc}}$ & $40.87^{\mathrm{b}}$ & $24.50^{\mathrm{ab}}$ & Red & Orange & High \\
AU-9 & $38.55^{\mathrm{bc}}$ & $33.67^{\mathrm{cd}}$ & $21.18^{\mathrm{bc}}$ & Light red & Light orange & Low \\
AU-10 & $41.40^{\mathrm{b}}$ & $40.02^{\mathrm{bc}}$ & $22.36^{\mathrm{bc}}$ & Red & Light orange & Low \\
AU-11 & $40.84^{\mathrm{bc}}$ & $44.67^{\mathrm{a}}$ & $28.55^{\mathrm{a}}$ & Dark red & Dark orange & Medium \\
AU-12 & $46.77^{\mathrm{a}}$ & $43.87^{\mathrm{ab}}$ & $26.30^{\mathrm{ab}}$ & Dark red & Orange & Low \\
AU-13 & $37.79^{\mathrm{bc}}$ & $36.18^{\mathrm{c}}$ & $21.90^{\mathrm{bc}}$ & Light red & Orange & High \\
AU-14 & $42.45^{\mathrm{ab}}$ & $41.11^{\mathrm{ab}}$ & $25.86^{\mathrm{ab}}$ & Dark red & Orange & Low \\
AU-15 & $40.04^{\mathrm{bc}}$ & $35.40^{\mathrm{cd}}$ & $22.07^{\mathrm{bc}}$ & Light red & Light orange & Low \\
AU-16 & $43.35^{\mathrm{ab}}$ & $34.27^{\mathrm{cd}}$ & $23.33^{\mathrm{bc}}$ & Light red & Light orange & Medium \\
\hline
\end{tabular}

Different letters in same column indicate significant $(p<0.05)$ differences among genotypes.

The harvest dates of genotypes were found between 2 November (AU-13) and 21 November (AU-6). Among the studied genotypes, the highest fruit weight was obtained from AU-11 genotype as $6.86 \mathrm{~g}$, and followed by AU-5 as $6.44 \mathrm{~g}$, while the lowest fruit weight was observed in genotype AU-4 as 3.89 g, respectively (Table 1). We found a two-fold difference between bigger and smaller fruit bearing genotypes (AU-11 and AU-4) (Table 1). In contrast to fruit weight, the highest number of fruits per cluster was seen in the AU-4 genotype as 6.74, whereas the lowest value was determined in the AU-11 genotype as 3.61. The shape index and pedicel length of fruits were between 0.78 and 1.09 and 2.93 and $5.81 \mathrm{~mm}$, respectively. The genotypes exhibited leaf length and leaf width between 32.15 and $51.22 \mathrm{~mm}$ and 14.33 and $27.30 \mathrm{~mm}$, respectively, indicating 1.5-fold and 2-fold differences for length and width among genotypes (Table 1).

Fruit color is one of the most appealing characters for commercial acceptance of any variety of horticultural crops. In the present study, fruit external $L, a$ and $b$ color index varied among genotypes from 37.56-46.77 for $L$ color indices, 32.23-44.67 for $a$ color indices and 20.27-28.55 for $b$ color indices, respectively (Table 2). Fruit peel color and flesh color were also found to be different among genotypes. Seven genotypes had light red fruit peel color, 5 genotypes had dark red, and four genotypes had red 
peel color. In addition, seven genotypes had orange flesh color, seven genotypes had light orange flesh color, and only two genotypes had dark orange flesh color (Table 2).

Table 3 shows organic acid concentration of 16 strawberry tree genotypes. The genotypes differed each other statistically in terms of malic acid and citric acid concentration $(p<0.05)$. However, there were no statistical differences among genotypes in terms of oxalic acid, succinic acid, and fumaric acid concentration (Table 3). The predominant organic acid in fruits of tested strawberry tree genotypes was malic acid (1.12-3.07 mg/100 g). Malic acid is followed by citric acid and citric acid concentration varied between $0.44-1.03 \mathrm{mg} / 100 \mathrm{~g}$. Strawberry tree fruits also included succinic acid $(0.22-0.46 \mathrm{mg} / 100 \mathrm{~g})$, fumaric acid $(0.17-0.40 \mathrm{mg} / 100 \mathrm{~g})$, respectively. Oxalic acid $(0.10-0.29 \mathrm{mg} / 100 \mathrm{~g})$ was present in lowest quantities compared to other analyzed acids.

Table 3. Organic acid concentration in fruits of strawberry tree genotypes (average of 2017-2018 years) (g/100 g FW).

\begin{tabular}{cccccc}
\hline Genotypes & Malic Acid & Citric Acid & Succinic Acid & Fumaric Acid & Oxalic Acid \\
\hline AU-1 & $3.07^{\mathrm{a}}$ & $0.60^{\mathrm{bc}}$ & $0.20^{\mathrm{NS}}$ & $0.28^{\mathrm{NS}}$ & $0.25^{\mathrm{NS}}$ \\
AU-2 & $2.44^{\mathrm{bc}}$ & $0.55^{\mathrm{bc}}$ & 0.24 & 0.40 & 0.20 \\
AU-3 & $3.02^{\mathrm{ab}}$ & $0.66^{\mathrm{bc}}$ & 0.46 & 0.30 & 0.25 \\
AU-4 & $1.29^{\mathrm{fg}}$ & $0.81^{\mathrm{ab}}$ & 0.33 & 0.35 & 0.29 \\
AU-5 & $1.56^{\mathrm{ef}}$ & $0.70^{\mathrm{bc}}$ & 0.30 & 0.22 & 0.22 \\
AU-6 & $2.05^{\mathrm{d}}$ & $0.50^{\mathrm{c}}$ & 0.22 & 0.30 & 0.15 \\
AU-7 & $2.83^{\mathrm{b}}$ & $0.44^{\mathrm{c}}$ & 0.25 & 0.19 & 0.18 \\
AU-8 & $1.50^{\mathrm{f}}$ & $0.91^{\mathrm{ab}}$ & 0.30 & 0.17 & 0.13 \\
AU-9 & $1.12^{\mathrm{g}}$ & $1.00^{\mathrm{ab}}$ & 0.40 & 0.35 & 0.10 \\
AU-10 & $1.20^{\mathrm{fg}}$ & $0.76^{\mathrm{b}}$ & 0.27 & 0.25 & 0.13 \\
AU-11 & $2.96^{\mathrm{ab}}$ & $0.85^{\mathrm{ab}}$ & 0.40 & 0.28 & 0.21 \\
AU-12 & $2.35^{\mathrm{c}}$ & $0.73^{\mathrm{b}}$ & 0.33 & 0.24 & 0.18 \\
AU-13 & $2.83^{\mathrm{b}}$ & $1.03^{\mathrm{a}}$ & 0.43 & 0.28 & 0.11 \\
AU-14 & $1.39^{\mathrm{fg}}$ & $0.80^{\mathrm{ab}}$ & 0.40 & 0.35 & 0.14 \\
AU-15 & $1.45^{\mathrm{fg}}$ & $0.70^{\mathrm{bc}}$ & 0.35 & 0.30 & 0.24 \\
AU-16 & $1.80^{\mathrm{e}}$ & $0.85^{\mathrm{ab}}$ & 0.30 & 0.20 & 0.20 \\
\hline
\end{tabular}

Different letters in same column indicate significant $(p<0.05)$ differences among genotypes. NS.: non significant. FW: fresh weight.

Statistically significant differences are evident for all specific sugars among genotypes at $p<0.05$ level (Table 4). The most abundant sugars were fructose $(6.09-10.56 \mathrm{~g} / 100 \mathrm{~g})$, followed by glucose $(3.85-6.07 \mathrm{~g} / 100 \mathrm{~g})$, and sucrose $(0.78-1.56 \mathrm{~g} / 100 \mathrm{~g})$ (Table 4).

The total phenolic concentration, total dietary fiber, total lipid, vitamin C, and total antioxidant capacities of 16 seed propagated strawberry tree fruits are presented in Table 5. All searched above parameters, indicating statistically significant differences $(p<0.05)$. Total phenolic concentration of genotypes were in descending order AU-12 (818 mg GAE/100 g) > AU-10 (804 mg GAE/100 g) > AU-5 $(776 \mathrm{mg}$ GAE$/ 100 \mathrm{~g})>\mathrm{AU}-11(744 \mathrm{mg} \mathrm{GAE} / 100 \mathrm{~g})>$ AU-2 $(728 \mathrm{mg} \mathrm{GAE} / 100 \mathrm{~g})>$ AU-15 $(717 \mathrm{mg}$ GAE/100 g) > AU-8 (703 mg GAE/100 g) > AU-3 (691 mg GAE/100 g) > AU-14 (680 mg GAE/100 g) > AU-16 (655 mg GAE/100 g) > AU-4 (641 mg GAE/100 g) > AU-9 (628 mg GAE/100 g) > AU-13 (607 mg GAE/100 g) > AU-7 (594 mg GAE/100 g) > AU-1 (584 mg GAE/100 g) > AU-6 (567 mg GAE/100 g), respectively. The highest total dietary fiber concentration was found in genotype AU-2 as $12.76 \mathrm{~g} / 100 \mathrm{~g}$ and the genotype AU-8 showed the lowest values as $7.04 \mathrm{~g} / 100 \mathrm{~g}$ and most of the genotypes such as AU-11 to AU-16 were statistically placed as the same group. Strawberry tree fruits revealed very low lipid concentration between $0.28-0.54 \mathrm{~g} / 100 \mathrm{~g}$ FW. The vitamin $\mathrm{C}$ and total antioxidant capacity were found quite variable among genotypes, which are in range of $58-93 \mathrm{mg} / 100 \mathrm{~g}$ FW and 16.30-29.83 $\mu \mathrm{mol}$ TE/g FW, respectively (Table 5). 
Table 4. Sugar concentration in the fruit of strawberry tree genotypes (average of 2017-2018 years) (g/100 g FW).

\begin{tabular}{cccc}
\hline Genotypes & Fructose & Glucose & Sucrose \\
\hline AU-1 & $9.88^{\mathrm{ab}}$ & $4.11^{\mathrm{ef}}$ & $1.04^{\mathrm{bc}}$ \\
AU-2 & $8.67^{\mathrm{bc}}$ & $4.27^{\mathrm{ef}}$ & $1.15^{\mathrm{bc}}$ \\
AU-3 & $10.12^{\mathrm{ab}}$ & $5.42^{\mathrm{bc}}$ & $1.56^{\mathrm{a}}$ \\
AU-4 & $7.43^{\mathrm{cd}}$ & $4.45^{\mathrm{e}}$ & $1.41^{\mathrm{ab}}$ \\
AU-5 & $8.80^{\mathrm{bc}}$ & $3.85^{\mathrm{f}}$ & $0.89^{\mathrm{bc}}$ \\
AU-6 & $10.56^{\mathrm{a}}$ & $5.69^{\mathrm{b}}$ & $1.38^{\mathrm{ab}}$ \\
AU-7 & $7.24^{\mathrm{cd}}$ & $4.87^{\mathrm{d}}$ & $1.09^{\mathrm{bc}}$ \\
AU-8 & $8.06^{\mathrm{bc}}$ & $3.96^{\mathrm{ef}}$ & $1.22^{\mathrm{b}}$ \\
AU-9 & $8.40^{\mathrm{bc}}$ & $6.07^{\mathrm{a}}$ & $0.85^{\mathrm{bc}}$ \\
AU-10 & $9.08^{\mathrm{b}}$ & $4.00^{\mathrm{ef}}$ & $0.97^{\mathrm{bc}}$ \\
AU-11 & $7.55^{\mathrm{c}}$ & $6.02^{\mathrm{a}}$ & $1.50^{\mathrm{ab}}$ \\
AU-12 & $8.90^{\mathrm{bc}}$ & $5.89^{\mathrm{ab}}$ & $0.90^{\mathrm{bc}}$ \\
AU-13 & $6.09^{\mathrm{d}}$ & $5.44^{\mathrm{bc}}$ & $0.78^{\mathrm{c}}$ \\
AU-14 & $8.55^{\mathrm{bc}}$ & $5.02^{\mathrm{cd}}$ & $1.34^{\mathrm{ab}}$ \\
AU-15 & $9.21^{\mathrm{b}}$ & $4.58^{\mathrm{de}}$ & $1.28^{\mathrm{ab}}$ \\
AU-16 & $7.02^{\mathrm{cd}}$ & $5.25^{\mathrm{c}}$ & $1.19^{\mathrm{b}}$ \\
\hline
\end{tabular}

Different letters in the same column indicate significant $(p<0.05)$ differences among genotypes. FW: fresh weight.

Table 5. Total phenolic, total dietary fiber, total lipid, antioxidant capacity, and vitamin $\mathrm{C}$ content in the fruit of strawberry tree genotypes (average of 2017-2018 years).

\begin{tabular}{|c|c|c|c|c|c|}
\hline Genotypes & $\begin{array}{l}\text { Total Phenolic Content } \\
\text { (mg GAE per } 100 \text { g FW) }\end{array}$ & $\begin{array}{l}\text { Total Dietary Fibre } \\
\text { (g/100 g FW) }\end{array}$ & $\begin{array}{l}\text { Total Lipid } \\
\text { (g/100 g FW) }\end{array}$ & $\begin{array}{c}\text { Vitamin C } \\
(\mathrm{mg} / 100 \mathrm{~g} \text { FW) }\end{array}$ & $\begin{array}{c}\text { Antioxidant Capacity } \\
(\mu \mathrm{mol} T \mathrm{TE} / \mathrm{g} \text { FW })\end{array}$ \\
\hline AU-1 & 584 ef & $8.22^{b c}$ & $0.41^{\mathrm{ab}}$ & $62^{b c}$ & $18.11^{\mathrm{cd}}$ \\
\hline AU-2 & $728^{c}$ & $12.76^{\mathrm{a}}$ & $0.36^{a b}$ & $73^{b}$ & $27.02^{a b}$ \\
\hline AU-3 & $691^{\mathrm{cd}}$ & $12.07^{\mathrm{ab}}$ & $0.50^{\mathrm{ab}}$ & $58^{c}$ & $23.35^{b c}$ \\
\hline AU-4 & 641 de & $8.67^{b c}$ & $0.33^{a b}$ & $73^{b}$ & $20.18^{b c}$ \\
\hline AU-5 & $776^{\mathrm{b}}$ & $10.03^{b}$ & $0.28^{b}$ & $70^{b c}$ & $26.56^{\mathrm{ab}}$ \\
\hline AU-6 & $567^{\mathrm{f}}$ & $7.71^{b c}$ & $0.44^{\mathrm{ab}}$ & $82^{a b}$ & $16.30^{\mathrm{d}}$ \\
\hline AU-7 & $594^{\text {ef }}$ & $11.56^{\mathrm{ab}}$ & $0.30^{\mathrm{b}}$ & $73^{b}$ & $17.79^{\mathrm{cd}}$ \\
\hline AU-8 & $703^{\mathrm{cd}}$ & $7.04^{c}$ & $0.52^{\mathrm{ab}}$ & $65^{\mathrm{bc}}$ & $24.12^{b}$ \\
\hline AU-9 & $628^{\mathrm{e}}$ & $9.30^{b c}$ & $0.50^{a b}$ & $90^{\mathrm{ab}}$ & $21.55^{b c}$ \\
\hline AU-10 & $804^{\mathrm{ab}}$ & $10.40^{\mathrm{ab}}$ & $0.33^{a b}$ & $87^{a b}$ & $28.15^{a b}$ \\
\hline AU-11 & $744^{b c}$ & $7.98^{\mathrm{bc}}$ & $0.47^{a b}$ & $77^{a b}$ & $26.04^{a b}$ \\
\hline AU-12 & $818^{a}$ & $8.04^{b c}$ & $0.54^{\mathrm{a}}$ & $93^{a}$ & $29.83^{a}$ \\
\hline AU-13 & 607 ef & $9.54^{b c}$ & $0.37 \mathrm{ab}$ & $62 \mathrm{bc}$ & $18.76^{\mathrm{c}}$ \\
\hline AU-14 & $680^{d}$ & $8.85^{b c}$ & $0.43^{a b}$ & $85^{\mathrm{ab}}$ & $25.28^{\mathrm{ab}}$ \\
\hline AU-15 & $717^{\mathrm{cd}}$ & $9.02 \mathrm{bc}$ & $0.40^{a b}$ & $69 \mathrm{bc}$ & $25.04^{a b}$ \\
\hline AU-16 & 655 de & $9.83^{b c}$ & $0.36^{a b}$ & $60^{b c}$ & $22.20^{b c}$ \\
\hline
\end{tabular}

Different letters in same column indicate significant $(p<0.05)$ differences among genotypes. FW: fresh weight.

Among the identified phenolic acids, four major phenolic acids were determined and given in Table 6. Among them, gallic acid was the most representative, ranging from 3.04 to $9.27 \mathrm{mg} / 100 \mathrm{~g}$. The highest amount was found in AU-12 as $9.27 \mathrm{mg} / 100 \mathrm{~g}$ and followed by AU-10 as $8.94 \mathrm{mg} / 100 \mathrm{~g}$. Catechin was significantly higher in the AU-12 genotype with a value of $6.84 \mathrm{mg} / 100 \mathrm{~g}$, whereas AU-6 genotype had significantly lower values as $2.21 \mathrm{mg} / 100 \mathrm{~g}$. The measured chlorogenic and ellagic acid derivative ranged from 1.44 to $4.07 \mathrm{mg} / 100 \mathrm{~g}$ and $1.28-3.02 \mathrm{mg} / 100 \mathrm{~g}$ (Table 6).

Syringic acid, rutin, phloridzin, vanilic acid, protocatechuie acid, $p$-coumaric acid, ferulic acid, and quercetin were the minor phenolic acids in strawberry tree fruits (Table 7). There was statistically significant difference among genotypes for some minor phenolic acids such as syringic acid, rutin, phloridzin, and vanilic acid $(p<0.05)$. However, protocatechuie acid, $p$-coumaric acid, ferulic acid, and quercetin were found not significant among genotypes (Table 7). 
Table 6. Major phenolic acids of strawberry tree genotypes (average of 2017-2018 years) (mg/100 g FW).

\begin{tabular}{ccccc}
\hline Genotypes & Gallic Acid & Catechin & Chlorogenic Acid & Ellagic Acid \\
\hline AU-1 & $3.27^{\mathrm{de}}$ & $2.86^{\mathrm{e}}$ & $1.44^{\mathrm{d}}$ & $1.54^{\mathrm{bc}}$ \\
AU-2 & $7.74^{\mathrm{b}}$ & $5.35^{\mathrm{bc}}$ & $3.10^{\mathrm{b}}$ & $2.32^{\mathrm{ab}}$ \\
AU-3 & $6.10^{\mathrm{cd}}$ & $4.91^{\mathrm{c}}$ & $2.85^{\mathrm{bc}}$ & $1.41^{\mathrm{bc}}$ \\
AU-4 & $4.82^{\mathrm{d}}$ & $3.50^{\mathrm{de}}$ & $2.56^{\mathrm{bc}}$ & $1.60^{\mathrm{bc}}$ \\
AU-5 & $8.42^{\mathrm{ab}}$ & $6.51^{\mathrm{ab}}$ & $3.70^{\mathrm{ab}}$ & $3.02^{\mathrm{a}}$ \\
AU-6 & $3.04^{\mathrm{e}}$ & $2.21^{\mathrm{ef}}$ & $1.58^{\mathrm{cd}}$ & $2.35^{\mathrm{ab}}$ \\
AU-7 & $3.65^{\mathrm{de}}$ & $2.52^{\mathrm{ef}}$ & $1.50^{\mathrm{cd}}$ & $1.28^{\mathrm{c}}$ \\
AU-8 & $6.31^{\mathrm{c}}$ & $4.72^{\mathrm{cd}}$ & $1.70^{\mathrm{cd}}$ & $2.04^{\mathrm{bc}}$ \\
AU-9 & $4.45^{\mathrm{de}}$ & $3.85^{\mathrm{d}}$ & $1.94^{\mathrm{cd}}$ & $1.80^{\mathrm{bc}}$ \\
AU-10 & $8.94^{\mathrm{ab}}$ & $5.92^{\mathrm{b}}$ & $4.07^{\mathrm{a}}$ & $2.70^{\mathrm{ab}}$ \\
AU-11 & $7.56^{\mathrm{bc}}$ & $6.20^{\mathrm{ab}}$ & $3.32^{\mathrm{ab}}$ & $1.50^{\mathrm{bc}}$ \\
AU-12 & $9.27^{\mathrm{a}}$ & $6.84^{\mathrm{a}}$ & $3.95^{\mathrm{ab}}$ & $2.81^{\mathrm{ab}}$ \\
AU-13 & $4.04^{\mathrm{de}}$ & $3.17^{\mathrm{de}}$ & $1.80^{\mathrm{cd}}$ & $2.24^{\mathrm{ab}}$ \\
AU-14 & $5.85^{\mathrm{cd}}$ & $4.33^{\mathrm{cd}}$ & $2.40^{\mathrm{bc}}$ & $1.73^{\mathrm{bc}}$ \\
AU-15 & $6.84^{\mathrm{bc}}$ & $5.12^{\mathrm{bc}}$ & $3.03^{\mathrm{bc}}$ & $2.15^{\mathrm{b}}$ \\
AU-16 & $5.67^{\mathrm{cd}}$ & $4.02^{\mathrm{cd}}$ & $2.11^{\mathrm{c}}$ & $1.46^{\mathrm{bc}}$ \\
\hline
\end{tabular}

Different letters in same column indicate significant $(p<0.05)$ differences among genotypes. FW: fresh weight.

Table 7. Minor phenolic compounds of strawberry tree genotypes (average of 2017-2018 years) $(\mathrm{mg} / 100 \mathrm{~g} \mathrm{FW})$.

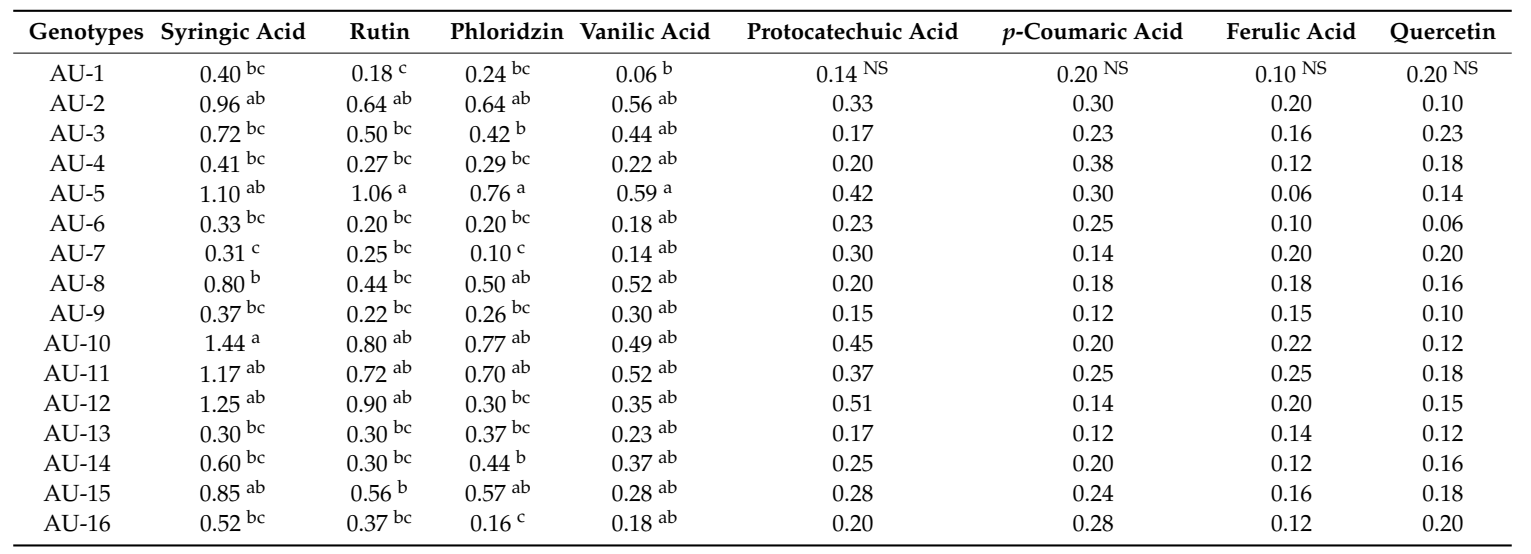

Different letters in same column indicate significant $(p<0.05)$ differences among genotypes NS: non significant. FW: fresh weight.

According to PCA analysis, harvest date, fruit weight, the number of fruit per cluster, total phenolic content, vitamin C, and dietary fiber content were found to be the most influential variables. Therefore, they were deemed an important component in the selection of genotypes. PCA helps in selecting the genotype with the desired characteristics. Two separate groups were obtained mainly based of the above discriminative traits and the AU-10 genotype placed between two groups (Figure 1). The first group (cluster 1) included AU-11, AU-15, AU-2, AU-3, AU-14, AU-07, AU-05, and AU-08 genotypes and characterized by bigger fruits, the lowest fruit number per cluster, high total phenolic content, and high antioxidant capacity. Dark orange flesh colored genotypes were also found in cluster 1. Cluster 2 was characterized by high sugar content. By using morphological and biochemical features together, $73.44 \%$ of the variability observed in the traits and the first three components namely PC1, PC2, and PC3 explained variability $41.40 \%, 23.02 \%$, and $9.00 \%$, respectively. The PCA figure clearly indicates high diversity among wild grown strawberry tree genotypes. The correlation coefficients between fruit traits of 16 strawberry tree genotypes were carried out (data are not shown). Fruit weight showed a significant negative correlation to the number of fruits per cluster $(\mathrm{r}=-0.88 ; p<0.01)$, indicating heavier clusters gave smaller fruits. Fruit weight was also negatively correlated with fructose concentrations $(r=-0.74, p<0.01)$ and revealed that larger fruits had lower tasting quality. There was a positive correlation among harvest date and fructose concentrations $(\mathrm{r}=0.85 ; p<0.01)$. In other 
words, late harvested fruits gave higher sugar content. Total phenolic content and antioxidant capacity were also positively correlated $(\mathrm{r}=0.87 ; p<0.01)$. Moreover, there was a moderate positive correlation between vitamin $C$ and antioxidant capacity values $(r=0.54, p<0.01)$.

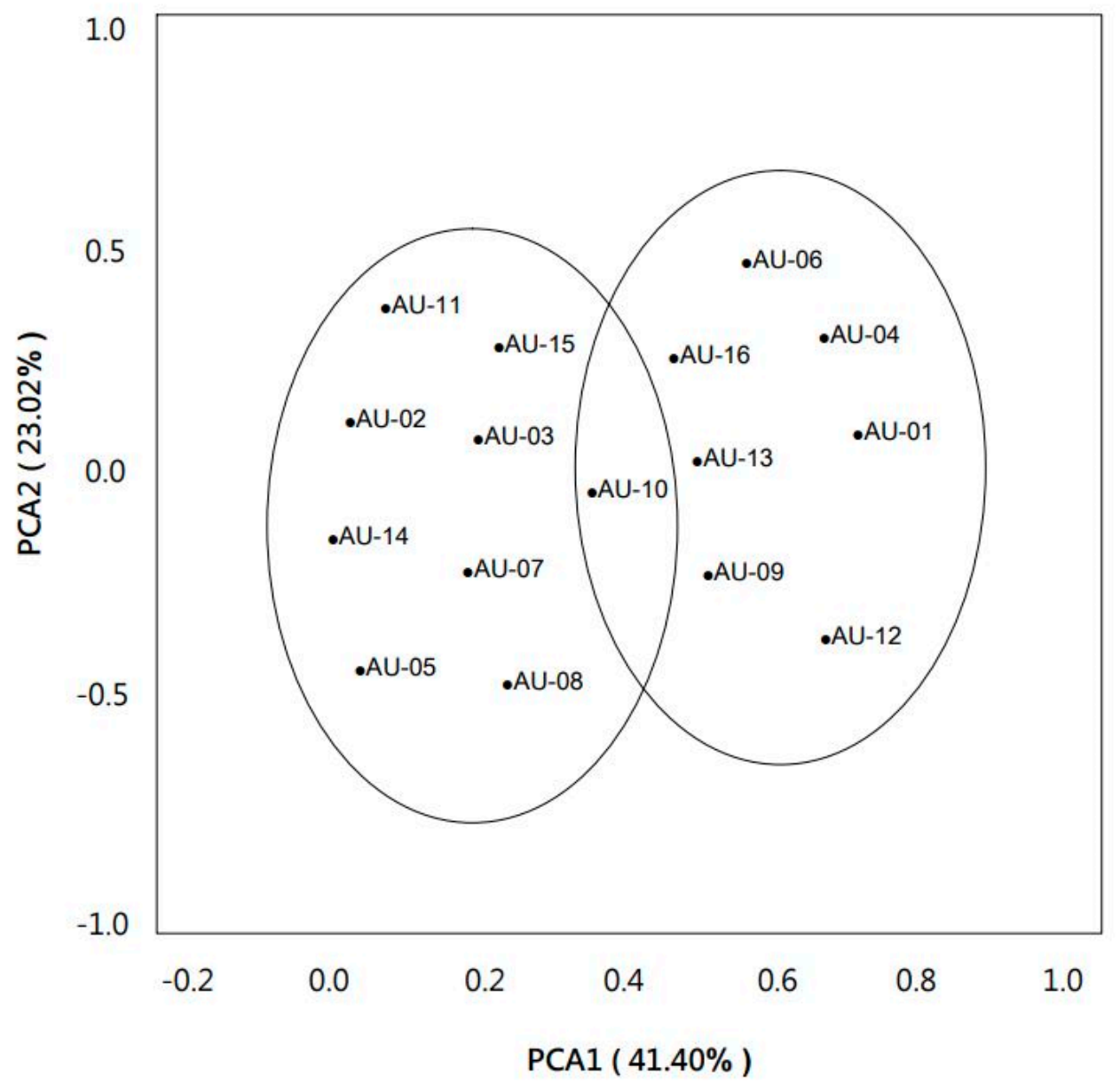

Figure 1. Scatter plot graphs showing the relationships between 16 individual strawberry tree genotypes based on morphological and biochemical data.

\section{Discussion}

In the present study, 16 strawberry tree genotypes showed considerable variation on harvest date, fruit weight, the number of fruit per cluster, shape index, fruit pedicel length, leaf length and width, vitamin $C$, total phenolic, antioxidant capacity, dietary fiber, and phenolic acids. This high variation could be explained on the basis of the difference in the genotypic background of the strawberry tree, which shows rich genetic diversity arising from natural seed propagation in the Black Sea region. In fact, long-term seed propagation and also human selection revealed great diversity of strawberry tree plants in different parts of Turkey [25,33-35].

Harvest dates of seed propagated strawberry tree genotypes grown in different agro-climatic conditions in Turkey have been reported between the end of October and at the beginning of December and notably, early harvested fruit receive appreciably high prices on the market [33,36,37].

High variability of the fruit weight, fruit shape, fruit pedicel length, and plant leaf dimensions of strawberry tree plants are evident. Fruit weight of different seed propagated strawberry tree genotypes grown in different coastal parts of the Turkey have been reported between 2.33-11.08 g [6,25,34,35,37-40]. Fruit breeding programs carried out in different parts of the world focused on a wide range of specific objectives such as increased cold hardiness, disease resistance, fruit quality, etc. However, all commercial scion releases must have high yield, bigger fruits, and quality 
fruit [41-43]. Appearance is one of the main components of the fruit quality trait. The characteristics that affect the appearance are primarily size and color. Consumers in general are willing to pay more to make larger fruit more profitable and fruit size has a large genetic component, thus selecting for larger fruit is relatively straight-forward [44]. Thus, some of the promising genotypes such as AU-2, AU-5, AU-11, and AU-14 that have relatively bigger fruits $(>6.0 \mathrm{~g})$, along with high yield, indicate a priority to register them as cultivar to increase popularity of this fruit.

The number of fruits per cluster, shape index, and fruit pedicel length of genotypes were found to be quite variable. Genetic variability in breeding materials is essential for a successful plant breeding program and understanding the magnitude of variability in crop species is pivotal since it provides the foundation for selection. The number of fruits per cluster was reported in the range of 3.20-8.60 among strawberry fruit genotypes [25,37,40]. The fruit shape index was in the range of 0.67-1.42 [34,35,38-40,45]. Toy [40] used 37 strawberry tree genotypes in the middle Black Sea region and reported fruit pedicel length, leaf length, and leaf width between $3.58-6.17 \mathrm{~mm}, 35.32-61.43 \mathrm{~mm}$, and $13.29-30.00 \mathrm{~mm}$, respectively indicating similarity with our study.

As indicated before, appearance is one of the main components of fruit quality trait. Variation in peel and flesh color among genotypes may be explained on the basis of difference in seed propagated nature of the genotypes. $L, a$, and $b$ color indices were reported on seed propagated strawberry tree genotypes in the range of 30.20-48.72, 24.62-45.22, and 19.81-36.51, respectively [25,37,39,40]. Toy [40]. reported that 37 strawberry tree genotypes in Unye and Fatsa districts in the Black Sea region had equal dark and light red peel color and dark and light orange fruit flesh color. Peel roughness is another important morphological parameter and nine of our genotypes had low roughness, five genotypes had medium, and two genotypes had high roughness. Kocabas [36] used 40 strawberry tree genotypes in Yalova province and reported that most of the genotypes had medium or low roughness on peel.

Malic acid was dominant in fruits of our 16 strawberry tree genotypes, followed by citric acid. Organic acids are of great significance in horticulture crops. In most of the fruit species, citric, malic, and tartaric acids are major organic acids and they are involved in quality keeping of the product obtained from fruits. Fruit maturation, senescence, tart taste, freshness, etc., are strongly affected by organic acid content $[46,47]$. Malic and citric acid has been reported as the main organic acid in strawberry tree fruits [48] that contributes to the pleasantly sour taste of fruits, and it is used as a food additive. Plant genotype determines organic acid content of fruits $[49,50]$ and environmental factors; horticultural practices including irrigation, pruning, soil type, etc., also influence organic acid content [51].

Fructose content in fruits of 16 strawberry tree genotypes is 2-fold higher than glucose and 8 -fold higher than sucrose. Previous studies also indicated that strawberry trees are rich in fructose, followed by glucose and sucrose. The fructose, glucose, and sucrose content were reported between $0.02-1.93 ; 2.32-8.34$, and 3.08-17.93 g/100 g in Spain and Turkey $[23,38,39]$, which is in accordance with our results. Boussalah et al. [45] reported that fructose and glucose constitute the total soluble sugars for all samples of wild grown strawberry trees grown in Algeria and varied from 5.52-8.44 and $2.90-5.24 \mathrm{~g} / 100 \mathrm{~g}$, respectively.

The 16 strawberry tree genotypes exhibited total phenolic content between 567-818 mg GAE/100 g FW. Our values of total phenolic content were in line with generally accepted values previously found in strawberry tree fruits grown in Spain, Croatia, and Turkey, 483 to $1973 \mathrm{mg}$ GAE/100 g FW, respectively $[23,37,48]$. The local strawberry tree genotypes are characterized by markedly higher contents of total polyphenols compared to blackberry (262 mg/100 g), blueberry (300 mg/100 g), raspberry (322 mg/100 g), strawberry (323 mg/100 g), and blackcurrant (434 mg/100 g) [52].

Our strawberry tree genotypes showed high dietary fiber content between 7.04-12.76 g/100 g FW. Previous studies also indicated the richness of strawberry tree fruits for dietary fiber, ranging 9.28-22.20 g/100 g [23,37,45]. According to these results, and despite natural variations, fiber content in fruits of strawberry trees grown in Turkey fruits was always over $6 \mathrm{~g} / 100 \mathrm{~g}$, indicating that it could be considered as an excellent source of fiber for human populations, which is of great importance in light 
of the fact that modern diets are often lacking dietary fiber, and that Occidental diets are nowadays lacking in dietary fiber. The importance of fiber for the normal function of the digestive system has been long appreciated. Considering the recommendation of fiber intake, which is $21-38 \mathrm{~g} /$ day for adults [53], the intake of $100 \mathrm{~g}$ of strawberry-tree fruits could provide $30-40 \%$ of the daily recommendation of fiber. According to European Regulations, A. unedo fruits could be considered under the approved mention "high in fiber" [54].

Total lipid content of all strawberry tree fruits was relatively low changed, from 0.28 to $0.54 \mathrm{~g} / 100 \mathrm{~g}$. Total lipid content in strawberry tree genotypes was reported $0.35-0.89 \mathrm{~g} / 100 \mathrm{~g}$ in different Mediterranean countries $[37,45,55]$, which indicate similarities with our findings.

The vitamin C content was affected by 16 strawberry tree genotypes and varied from 58 to $93 \mathrm{mg} / 100 \mathrm{~g}$, indicating richness of vitamin $C$ in strawberry tree fruits. When compared to some common fruits, vitamin $C$ content of strawberry trees is higher than cherry laurel $(5.28 \mathrm{mg} / 100 \mathrm{~g})$, blueberry (12.69 mg/100 g), raspberry ( $31.93 \mathrm{mg} / 100 \mathrm{~g})$, blackberry $(45.07 \mathrm{mg} / 100 \mathrm{~g})$, and strawberry $(57.95 \mathrm{mg} / 100 \mathrm{~g})$ but equal or higher than cornelian cherry $(50 \mathrm{mg} / 100 \mathrm{~g})[13,52,56]$. In fact, strawberry tree fruits show great genotypic differences for vitamin C. It was reported between $98-280 \mathrm{mg} / 100 \mathrm{~g}$ [6], $13-126 \mathrm{mg} / 100 \mathrm{~g}$ [39], 47-59 mg/100 g [38], 46-60 mg/100 g [37] and 93-203 mg/100 g [23]. Vitamin C content mainly determined by genotype but harvest date, growing location, rootstock, etc., also had significant effect on the amount of vitamin $C$ [23]. Consumption of vitamin $C$ rich fruits might have a beneficial effect on health, including protection against cardiovascular diseases and assisting in the proper functioning of the immune system [57].

Total antioxidant capacity (TAC) determined by the TEAC method on the fruits of 16 strawberry tree genotypes showed variation from 16.30 to 29.83 , indicating nearly 2 -fold differences between genotypes that have the lowest and the highest TAC values. Gundogdu et al. [38] found TAC among strawberry tree genotypes between 17.51 and $30.06 \mu \mathrm{mol} \mathrm{TE} / \mathrm{g}$, respectively, indicating similarities with our study. Colak [37] also found high TAC values among strawberry tree genotypes (18.07-33.40 $\mu \mathrm{mol}$ $\mathrm{TE} / \mathrm{g}$ ). Antioxidant activity was widely studied on strawberry tree fruits by using different antioxidant determining methods such as TEAC, FRAP, DPPH, etc., and all studies indicated that strawberry tree fruits had high antioxidant activity and antioxidant activity found to be genotype dependent. Moreover, the studies indicated that the type of extraction of phenols present in fruits of the strawberry tree also influenced the antioxidant activity [23,33,55,58-62].

Our samples had gallic acid, catachin, chlorogenic acid, and ellagic acid as the main phenolic acids. Gundogdu et al. [38] reported gallic acid, catechin, chlorogenic acid, and ellagic acid between $1.62-7.29 \mathrm{mg} / 100 \mathrm{~g}, 1.16-5.75 \mathrm{mg} / 100 \mathrm{~g}, 1.23-3.14 \mathrm{mg} / 100 \mathrm{~g}$, and $1.11-2.13 \mathrm{mg} / 100 \mathrm{~g}$, respectively among 8 seed propagated strawberry tree genotypes grown in western Turkey, which indicated agreement with our present findings.

\section{Conclusions}

Strawberry tree fruits remain rather unexploited regarding their horticultural properties, since relatively few studies exist concerning their socio-economic and health-promising benefits. The results showed an increased diversity of strawberry tree fruits for mainly local use but also possible use for other market. The results also indicated that local genotypes could be important as natural sources for human health content. In addition, development of strawberry tree fruits using diverse genotypes-based horticultural characteristics and ingredients should focus on resource efficiency, environmentally friendly, and innovative solutions. The present work will lead the food and pharmaceutical industries to consider strawberry tree fruits for the development of value-added functional food and medicinal supplements, as well hence areas that appear highly valuable to exert socioeconomic impact on the country. 
Author Contributions: Conceptualization, H.I.S., G.I. and S.E.; data curation, H.I.S., G.I. and S.E.; formal analysis, H.I.S., G.I. and S.E.; methodology, H.I.S., G.I. and S.E.; project administration, S.E.; visualization, M.A.A., H.Z., H.H., T.N., I.O.; writing—original draft, M.A.A., H.Z., H.H., T.N., I.O. and S.E.; writing—review and editing, M.A.A., H.Z., H.H., T.N., I.O. and S.E. All authors have read and agreed to the published version of the manuscript.

Funding: This research received no external funding and APC was funded by Mendel University.

Acknowledgments: The cooperation and research were supported of the program no. 6.2.10 ref.: 51834/2017-MZE17253, sub-program" National Program of Conservation and Utilization of Plant Genetic Resources and Agrobiodiversity" which are funded by the Ministry of Agriculture of the Czech Republic.

Conflicts of Interest: The authors declare that they have no conflict of interest.

\section{References}

1. Food and Agricultural Organization. 2019. Available online: http://www.fao.org/faostat (accessed on 26 May 2020).

2. Serce, S.; Ozgen, M.; Torun, A.A.; Ercisli, S. Chemical composition, antioxidant activities and total phenolic content of Arbutus andrachne L. (Fam. Ericaceae) (the Greek strawberry tree) fruits from Turkey. J. Food Compos. Anal. 2010, 23, 619-623. [CrossRef]

3. Gecer, M.K.; Kan, T.; Gundogdu, M.; Ercisli, S.; Ilhan, G.; Sagbas, H.I. Physicochemical characteristics of wild and cultivated apricots (Prunus armeniaca L.) from Aras valley in Turkey. Genet. Resour. Crop Evol. 2020, 67, 935-945. [CrossRef]

4. Serdar, U.; Mercan, L.; Okumus, A.; Soylu, A. Morphological and molecular comparison of selected chestnut (Castanea sativa Mill.) genotypes from black sea region of Turkey. Anadolu J. Agric. Sci. 2014, 29, 54-62. [CrossRef]

5. Akbulut, M.; Ercisli, S.; Jurikova, T.; Mlcek, J.; Gozlekci, S. Phenotypic and bioactive diversity on medlar fruits (Mespilus germanica L.). Erwerbs-Obstbau 2016, 3, 185-191. [CrossRef]

6. Vavilov, N.I. Centres of Origin of Cultivated Plants. Bull. Appl. Bot. Genet. Plant Breed. 1926, 16, 1-248.

7. Zohary, D. Centers of diversity and centers of origin. In Genetic Resources in Plants-Their Exploration and Conservation; Frankel, O.H., Bennett, E., Eds.; Blackwell Science Publishing: Oxford, UK, 1970; pp. $33-42$.

8. Celikel, G.; Demirsoy, L.; Demirsoy, H. The strawberry tree (Arbutus unedo L.) selection in Turkey. Sci. Hortic. 2008, 118, 115-119. [CrossRef]

9. Ercisli, S.; Orhan, E.; Esitken, A.; Yildirim, N.; Agar, G. Relationships among some cornelian cherry genotypes (Cornus mas L.) based on RAPD analysis. Genet. Resour. Crop Evol. 2008, 55, 613-618. [CrossRef]

10. Ozturk, M.; Yucel, E.; Yarci, C.; Celik, A.; Aksoy, A. Plant diversity in the Turkish Black Sea Region and Strategies for its Conservation. In Conservation of the Biological Diversity as a Prerequisite for Sustainable Development in the Black Sea Region; NATO ASI Series (Series 2: Environmental Security); Kotlyakov, V., Upperbrink, M., Metreveli, V., Eds.; Springer: Dordrecht, The Netherlands, 1998; Volume 46.

11. Ozbucak, T.B.; Kutbay, H.G.; Akcin, O.E. The Contributıon of wild edible plants to human nutrition in the Black Sea region of Turkey. Ethnobot. Leafl. 2006, 1, 10.

12. Ozen, T. Antioxidant activity of wild edible plants in the Black Sea Region of Turkey. Grasas Y Aceites 2010, 61, 86-94. [CrossRef]

13. Ercisli, S.; Yilmaz, S.O.; Gadze, J.; Dzubur, A.; Hadziabulic, S.; Aliman, J. Some fruit characteristics of conelian cherry (Cornus mas L.). Not. Bot. Horti Agrobot. 2011, 39, 255-259. [CrossRef]

14. Cesoniene, L.; Daubaras, R.; Vencloviene, J.; Viskelis, P. Biochemical and agro-biological diversity of Viburnum opulus genotypes. Cent. Eur. J. Biol. 2010, 6, 864-871.

15. Cesoniene, L.; Daubaras, R.; Viskelis, P.; Sarkinas, A. Determination of the total phenolic and anthocyanin contents and antimicrobial activity of Viburnum opulus fruit juice. Plant Food Hum. Nutr. 2012, 67, 256-261. [CrossRef] [PubMed]

16. Mikulic-Petkovsek, M.; Schmitzer, V.; Slatnar, A.; Stampar, F.; Veberic, R. Composition of sugars, organic acids, and total phenolics in 25 wild or cultivated berry species. J. Food Sci. 2012, 77, C1064-C1070. [CrossRef]

17. Mikulic-Petkovsek, M.; Ivancic, A.; Todorovic, B.; Veberic, R.; Stampar, F. Fruit phenolic composition of different elderberry species and hybrids. J. Food Sci. 2015, 80, C2180-C2190. [CrossRef] [PubMed] 
18. Maeda, H.; Akagi, T.; Tao, R. Quantitative characterization of fruit shape and its differentiation pattern in diverse persimmon (Diospyros kaki) cultivars. Sci. Hortic. 2018, 228, 41-48. [CrossRef]

19. Ozkan, G.; Ercisli, S.; Zeb, A.; Agar, G.; Sagbas, H.I.; Ilhan, G.; Gundogdu, M. Some morphological and biochemical characteristics of wild grown Caucasian Whortleberry (Vaccinium arctostaphylos L.) genotypes from Northeastern Turkey. Not. Bot. Hortic. Agrobot. 2019, 47, 378-383. [CrossRef]

20. Cañas-Gutiérrez, G.P.; Alcaraz, L.; Hormaza, J.I.; Arango-Isaza, R.E.; Saldamando-Benjumea, C.I. Diversity of avocado (Persea Americana Mill.) cultivars from Antioquia (Northeast Colombia) and comparison with a worldwide germplasm collection. Turk. J. Agric. For. 2019, 43, 437-449. [CrossRef]

21. Zivotic, A.; Micic, N.; Zabic, M.; Bosancic, B.; Cvetkovic, M. Precision cane meristem management can influence fruit quality of floricane red raspberry cultivars. Turk. J. Agric. For. 2019, 43, 405-413. [CrossRef]

22. Chiorato, A.F.; Carbonell, S.A.M.; Benchimol, L.L.; Chiavega, T.O.M.B.; Dias, L.A.S.; Colombo, C.A. Genetic diversity in common bean accessions evaluated by means of morpho-agronomical and RAPD data. Sci. Agric. 2007, 64, 256-262. [CrossRef]

23. Ruiz-Rodríguez, B.M.; Morales, P.; Fernández-Ruiz, V.; Sánchez-Mata, M.C.; Cámara, M.; Díez-Marqués, C.; Pardo-De-Santayana, M.; Molina, M.; Tardío, J. Valorization of wild strawberry tree fruits (Arbutus unedo L.) through nutritional assessment and natural production data. Food Res. Int. 2011, 44, 1244-1253. [CrossRef]

24. McGuire, R.G. Reporting of objective color measurements. HortScience 1992, 27, 1254-1255. [CrossRef]

25. Islam, A.; Pehlivan, N.F. Pomological properties of strawberry tree (Arbutus unedo) grown in Marmara island. Acad. Agric. J. 2016, 5, 13-20.

26. Bevilacqua, A.E.; Califano, A.N. Determination of organic acids in dairy products by high performance liquid chromatography. J. Food Sci. 1989, 54, 1076-1079. [CrossRef]

27. Melgarejo, P.; Salazar, D.M.; Artes, F. Organic acids and sugars composition of harvested pomegranate fruits. Eur. Food Res. Technol. 2000, 211, 185-190. [CrossRef]

28. Ozgen, M.; Reese, R.N.; Tulio, A.Z.; Scheerens, J.C.; Miller, A.R. Modified 2.2- azino-bis-3ethylbenzothiazoline-6-sulfonic acid (ABTS) method to measure antioxidant capacity of selected small fruits and a comparison to ferric reducing antioxidant power (FRAP) and 2.2-diphenyl-1-picrylhdrazyl (DPPH) methods. J. Agric. Food Chem. 2006, 54, 1151-1157. [CrossRef]

29. Singleton, V.L.; Orthofer, R.; Lamuela-Raventos, R.M. Analysis of total phenols and other oxidation substrates and antioxidants by means of Folin-Ciocalteu reagent. Methods Enzymol. 1999, 299, 152-178.

30. De Padua, M.; Fontoura, P.S.G.; Mathias, A.L. Chemical composition of Ulvaria oxysperma (Kützing) bliding, Ulva lactuca (Linnaeus) and Ulva fascita (Delile). Braz. Arch. Biol. Technol. 2004, 47, 49-55. [CrossRef]

31. AOAC. Official Methods of Analysis of AOAC International, 16th ed.; AOAC International: Gaithersburg, MD, USA, 1999.

32. Rodriguez-Delgado, M.A.; Malovana, S.; Perez, J.P.; Borges, T.; Garcia-Montelongo, F.J. Separation of phenolic compounds by high- performance liquid chromatography with absorbance and fluorimetric detection. J. Chromatogr. 2001, 912, 249-257. [CrossRef]

33. Şeker, M.; Toplu, C. Determination and comparison of chemical characteristics of Arbutus unedo L. and Arbutus andrachnae L. (Family Ericaceae) fruits. J. Med. Food 2010, 13, 1013-1018. [CrossRef]

34. Zenginbal, H.; Gundogdu, M. Physicochemical characterization of strawberry tree (Arbutus unedo L.) genotypes naturally growing in Düzce and Zonguldak province. Anadolu J. Agric. Sci. 2016, 31, 332-336. [CrossRef]

35. Yarilgac, T.; Pekdemir, M. Promising strawberry tree genotypes from North Anatolia, Turkey. Erwerbs-Obstbau 2019, 61, 79-84. [CrossRef]

36. Kocabas, H. Selection of Strawberry Tree (Arbutus Unedo L.) Genotypes Naturally Grown in Yalova. Master's Thesis, Tokat Gaziosmanpaşa University Institute of Natural and Applied Sciences, Horticulture, Tokat, Turkey, 2019; p. 71.

37. Colak, A.M. Morphological and biochemical diversity in fruits of Arbutus unedo L. from East Aegean Region in Turkey. Erwerbs Obstbau 2019, 61, 379-383. [CrossRef]

38. Gundogdu, M.; Ercisli, S.; Canan, I.; Orman, E.; Sameeullah, M.; Naeem, M.; Ben Ayed, R. Diversity in phenolic compound, biochemical and pomological characteristics of Arbutus unedo fruits. Folia Hortic. 2018, 30, 139-146. [CrossRef] 
39. Cakmak, S. Determination of Some Biochemical Parameters of Arbutus (Arbutus spp.) Genotypes Grown in Bulancak District of Giresun Province. Master's Thesis, Institute of Natural and Applied Sciences, Horticulture, Ordu University, Ordu, Turkey, 2019; p. 53.

40. Toy, M. Determination of Some Pomological and Morphological Characteristics of Arbutus (Arbutus spp.) Genotypes Grown in Unye and Fatsa District. Master's Thesis, Institute of Natural and Applied Sciences, Horticulture, Ordu University, Ordu, Turkey, 2019; p. 48.

41. Ersoy, N.; Kupe, M.; Sagbas, H.I.; Ercisli, S. Phytochemical diversity among barberry (Berberis vulgaris L.). Not. Bot. Hortic. Sgrobot. 2018, 46, 198-204.

42. Colak, A.M.; Kupe, M.; Bozhuyuk, R.M.; Ercisli, S.; Gundogdu, M. Identification of some fruit characteristics in wild bilberry (Vaccinium myrtillus L.) accessions from Eastern Anatolia. Gesunde Pflanz. 2019, 70, 31-38. [CrossRef]

43. Ercisli, S.; Ipek, A.; Barut, E. SSR marker-based DNA fingerprinting and cultivar identification of olives (Olea europaea). Biochem. Genet. 2011, 49, 555-561. [CrossRef]

44. Janick, J.; Moore, J.N. (Eds.) Fruit Breeding. In Tree and Tropical Fruits; Wiley: New York, NY, USA, 1996; Volume I.

45. Boussalah, N.; Boussalah, D.; Cebadera-Miranda, L.; Fernández-Ruiz, V.; Barros, L.; Ferreira, I.C.F.R.; Cortes Sanchez Mata, M.; Madani, K. Nutrient composition of Algerian strawberry-tree fruits (Arbutus unedo L.). Fruits 2018, 73, 283-297. [CrossRef]

46. Saradhuldhat, P.; Paull, R.E. Pineapple organic acid metabolism and accumulation during fruit development. Sci. Hortic. 2007, 112, 297-303. [CrossRef]

47. Etienne, A.; Génard, M.; Lobit, P.; Mbeguié-A-Mbéguié, D.; Bugaud, C. What controls fleshy fruit acidity? A review of malate and citrate accumulation in fruit cells. J. Exp. Bot. 2013, 64, 1451-1469. [CrossRef]

48. Vidrih, R.; Hribar, J.; Prgomet, Z.; Poklar Ulrih, N. The physico-chemical properties of strawberry tree (Arbutus unedo L.) fruits. Croat. J. Food Sci. Technol. 2013, 5, 29-33.

49. Mikulic Petkovsek, M.; Stampar, F.; Veberic, R. Parameters of inner quality of the scab resistant and susceptible apple in organic and integrated production. Sci. Hortic. 2007, 114, 37-44. [CrossRef]

50. Wu, J.; Gao, H.; Zhao, L.; Liao, X.; Chen, F.; Wang, Z.; Hu, Z. Chemical composition of some apple cultivars. Food Chem. 2007, 103, 88-93. [CrossRef]

51. Hudina, M.; Stampar, F. Influence of frost damage on the sugars and organic acids contents in apple and pear flowers. Eur. J. Hortic. Sci. 2006, 71, 161-164.

52. Donno, D.; Cerutti, A.K.; Prgomet, I.; Mellano, M.G.; Beccaro, G.L. Foodomics for mulberry fruit (Morus spp.): Analytical fingerprint as antioxidants' and health properties' determination tool. Food Res. Int. 2015, 69, 179-188. [CrossRef]

53. Trumbo, P.; Schlicker, S.; Yates, A.A.; Poos, M. Dietary reference intakes for energy, carbohydrate, fiber, fat, fatty acids, cholesterol, protein, and amino acids. J. Am. Diet. Assoc. 2002, 102, 1621-1630. [CrossRef]

54. European Parliament and Council. Regulation (EU) No. 1169/2011 of the European Parliament and of the Council of 25 October 2011 on the provision of food information to consumers. Off. J. Eur. Union 2011, 304, 18-63.

55. Barros, L.; Carvalho, A.M.; Morais, J.S.; Ferreira, I.C.F.R. Strawberry-tree, blackthorn and rose fruits: Detailed characterization in nutrients and phytochemicals with antioxidant properties. Food Chem. 2010, 120, 247-254. [CrossRef]

56. Celik, F.; Ercisli, S.; Yilmaz, S.O.; Hegedus, A. Estimation of certain physical and chemical fruit characteristics of various cherry laurel (Laurocerasus officinalis Roem.) genotypes. HortScience 2011, 46, 924-927. [CrossRef]

57. Smirnoff, N. Ascorbic acid metabolism and functions: A comparison of plants and mammals. Free Radic. Biol. Med. 2018, 122, 116-129. [CrossRef]

58. Pallauf, K.; Rivas-Gonzalo, J.C.; Del Castillo, M.; Cano, M.P.; De Pascual-Teresa, S. Characterization of the antioxidant composition of strawberry tree (Arbutus unedo L.) fruits. J. Food Comp. Anal. 2008, 21, $273-281$. [CrossRef]

59. Fortalezas, S.; Tavares, L.; Pimpao, R.; Tyagi, M.; Pontes, V.; Alves, P.M.; McDougall, G.; Stewart, D.; Ferreira, R.B.; Santos, C.N. Antioxidant properties and neuroprotective capacity of strawberry tree fruit (Arbutus unedo). Nutrients 2010, 2, 214-229. [CrossRef] [PubMed] 
60. Mendes, L.; de Freitas, V.; Baptista, P.; Carvalho, P. Comparative antihemolytic and radical scavenging activities of strawberry tree (Arbutus unedo L.) leaf and fruit. Food Chem. Toxicol. 2011, 49, 2285-2291. [CrossRef] [PubMed]

61. Isbilir, S.S.; Orak, H.H.; Yagar, H.; Ekinci, N. Determination of antioxidant activities of strawberry tree (Arbutus unedo L.) flowers and fruits at different ripening stages. Acta Sci. Pol. Hortoru. 2012, 11, $223-237$.

62. Morales, P.; Ferreira, I.C.F.R.; Carvalho, A.M.; Fernández-Ruiz, V.; Sánchez-Mata, M.C.; Câmara, M.; Morales, R.; Tardio, J. Wild edible fruits as a potential source of phytochemicals with capacity to inhibit lipid peroxidation. Eur. J. Lipid Sci. Technol. 2013, 115, 176-185. [CrossRef]

Publisher's Note: MDPI stays neutral with regard to jurisdictional claims in published maps and institutional affiliations.

(C) 2020 by the authors. Licensee MDPI, Basel, Switzerland. This article is an open access article distributed under the terms and conditions of the Creative Commons Attribution (CC BY) license (http://creativecommons.org/licenses/by/4.0/). 